\title{
Extreme values of symmetric power $L$-functions at 1
}

\author{
by \\ Y.-K. LAU (Hong Kong) and J. Wu (Nancy)
}

1. Introduction. The study of the extreme values of Dirichlet $L$-functions at the point 1 has a long and rich history. Research on this topic was originated with a paper of Littlewood [15] in 1928 and was pursued by many authors (cf. [1], [2], [6], [7], [8], [23], [17] and [9]). A very good historical account can be found in [9], where Granville \& Soundararajan made important progress on the distribution of the extreme values of $L\left(1, \chi_{d}\right)$ for a real primitive character $\chi_{d}$ of modulus $|d|$.

Among the family of $L$-functions attached to the automorphic cuspidal representations for $\mathrm{GL}_{n}(\mathbb{Q})$ where $n \geq 1$, the Dirichlet $L$-functions constitute only a small part corresponding to $n=1$. The $\mathrm{GL}_{2}$ class consists of those $L$-functions associated to holomorphic cusp forms or Maass forms. The symmetric $m$ th power of a $\mathrm{GL}_{2} L$-function yields, under the Langlands functoriality conjecture if $m \geq 5$, an automorphic $\mathrm{GL}_{m+1} L$-function which is defined as an Euler product of degree $m+1$ (and thus called an $L$-function of degree $m+1$ ). The properties of these $L$-functions are of great current interest and their values at 1 are recently delved. Luo [16] investigated the case of symmetric square $L$-functions for Maass forms with large eigenvalue. Royer [18, 19], Habsieger \& Royer [10], Royer \& Wu [20] considered the first two symmetric power $L$-functions attached to holomorphic cusp forms with large squarefree level $\left({ }^{1}\right)$ while Cogdell \& Michel [3] and Royer \& Wu [21] considered all the symmetric power $L$-functions. Moreover, Lau \& Wu [14] studied similar problems in the weight aspect. In this paper we shall further study the extreme values of symmetric power $L$-functions at 1 .

Let us introduce our notation. For a positive even integer $k$, we denote by $\mathrm{H}_{k}^{*}(1)$ the set of all normalized Hecke primitive eigencuspforms of weight

2000 Mathematics Subject Classification: Primary 11F30, 11N36.

Key words and phrases: special values of automorphic $L$-series.

$\left({ }^{1}\right)$ An integer $n$ is called squarefree if $p^{2} \nmid n$ for all prime numbers $p$. 
$k$ for the modular group $\Gamma(1)=\mathrm{SL}_{2}(\mathbb{Z})$. It is a finite set with cardinality

$$
\left|\mathrm{H}_{k}^{*}(1)\right|=k / 12+O(1) .
$$

Here the normalization is such that the Fourier series expansion at the cusp $\infty$,

$$
f(z)=\sum_{n=1}^{\infty} \lambda_{f}(n) n^{(k-1) / 2} e^{2 \pi i n z} \quad(\operatorname{Im} z>0),
$$

has its first coefficient equal to one (i.e. $\lambda_{f}(1)=1$ ). Inherited from the Hecke operators, the Fourier coefficient $\lambda_{f}(n)$ satisfies the relation

$$
\lambda_{f}(m) \lambda_{f}(n)=\sum_{d \mid(m, n)} \lambda_{f}\left(\frac{m n}{d^{2}}\right)
$$

for all integers $m \geq 1$ and $n \geq 1$. According to Deligne [4], for any prime number $p$ there is a (complex) number $\alpha_{f}(p)$ such that

$$
\left|\alpha_{f}(p)\right|=1
$$

and

$$
\lambda_{f}\left(p^{\nu}\right)=\alpha_{f}(p)^{\nu}+\alpha_{f}(p)^{\nu-2}+\cdots+\alpha_{f}(p)^{-\nu}
$$

for all integers $\nu \geq 1$. Hence $\lambda_{f}(n)$ is a real multiplicative function of $n$.

Associated to each $f \in \mathrm{H}_{k}^{*}(1)$, the symmetric mth power L-function ( $m \in$ $\mathbb{N}$ ) is defined as

$$
L\left(s, \operatorname{sym}^{m} f\right):=\prod_{p} \prod_{0 \leq j \leq m}\left(1-\alpha_{f}(p)^{m-2 j} p^{-s}\right)^{-1}
$$

for $\sigma>1$; here and throughout, $\sigma$ and $\tau$ mean tacitly the real and imaginary parts of $s$, i.e. $s=\sigma+i \tau$. Multiplying out the Euler product, we see that it admits a Dirichlet series representation

$$
L\left(s, \operatorname{sym}^{m} f\right)=\sum_{n=1}^{\infty} \lambda_{\operatorname{sym}^{m} f}(n) n^{-s}
$$

for $\sigma>1$, where $\lambda_{\text {sym }^{m} f}(n)$ is a multiplicative function. By (1.4) and (1.6), we have for $n \geq 1$,

$$
\left|\lambda_{\text {sym }^{m} f}(n)\right| \leq \tau_{m+1}(n) .
$$

As is customary, $\tau_{m+1}(n)$ denotes the number of solutions in positive integers $n_{1}, \ldots, n_{m+1}$ of the equation $n=n_{1} \cdots n_{m+1}$. The case $m=1$ in (1.8) is commonly known as Deligne's inequality. For $m=1,2,3,4$, the symmetric power function $L\left(s, \operatorname{sym}^{m} f\right)$ can be analytically prolonged to $\mathbb{C}$ and satisfies the functional equation

$$
L_{\infty}\left(s, \operatorname{sym}^{m} f\right) L\left(s, \operatorname{sym}^{m} f\right)=\varepsilon\left(\operatorname{sym}^{m} f\right) L_{\infty}\left(1-s, \operatorname{sym}^{m} f\right) L\left(1-s, \operatorname{sym}^{m} f\right),
$$


where $\varepsilon\left(\operatorname{sym}^{m} f\right)= \pm 1$ and $L_{\infty}\left(s, \operatorname{sym}^{m} f\right)$ is the corresponding gamma factor (cf. [3, Section 1.1]).

In [14], Lau \& Wu proved the following results on the extreme values of $L\left(1, \operatorname{sym}^{m} f\right)$ in the weight aspect. Let $m=1,2,3,4$ and $2 \mid k$. For any $f \in \mathrm{H}_{k}^{*}(1)$, under $\mathrm{GRH}$ for $L\left(s, \operatorname{sym}^{m} f\right)$, we have

$$
\begin{aligned}
\{1+o(1)\}\left(2 B_{m}^{-} \log _{2} k\right)^{-A_{m}^{-}} & \leq L\left(1, \operatorname{sym}^{m} f\right) \\
& \leq\{1+o(1)\}\left(2 B_{m}^{+} \log _{2} k\right)^{A_{m}^{+}}
\end{aligned}
$$

as $k \rightarrow \infty$. In the opposite direction, it was shown unconditionally that there are $f_{m}^{ \pm} \in \mathrm{H}_{k}^{*}(1)$ such that for $k \rightarrow \infty$,

$$
\begin{aligned}
& L\left(1, \operatorname{sym}^{m} f_{m}^{+}\right) \geq\{1+o(1)\}\left(B_{m}^{+} \log _{2} k\right)^{A_{m}^{+}}, \\
& L\left(1, \operatorname{sym}^{m} f_{m}^{-}\right) \leq\{1+o(1)\}\left(B_{m}^{-} \log _{2} k\right)^{-A_{m}^{-}} .
\end{aligned}
$$

Here (and throughout) $\log _{j}$ denotes the $j$-fold iterated logarithm. The constants $A_{m}^{ \pm}$and $B_{m}^{ \pm}$are explicitly evaluated,

$$
\left\{\begin{array}{lll}
A_{m}^{+}=m+1, & B_{m}^{+}=e^{\gamma} & (m=1,2,3,4), \\
A_{m}^{-}=m+1, & B_{m}^{-}=e^{\gamma} \zeta(2)^{-1} & (m=1,3), \\
A_{2}^{-}=1, & B_{2}^{-}=e^{\gamma} \zeta(2)^{-2}, & \\
A_{4}^{-}=5 / 4, & B_{4}^{-}=e^{\gamma} B_{4}^{\prime-}, &
\end{array}\right.
$$

where $\zeta(s)$ is the Riemann zeta function, $\gamma$ denotes the Euler constant and $B_{4}^{\prime-}$ is a positive constant given by a rather complicated Euler product (cf. $[14,(1.16)])$.

The results in (1.9)-(1.11) determine completely, at least under GRH, the order of magnitude of $L\left(1, \operatorname{sym}^{m} f\right)$. Then it is interesting and natural to try to remove the assumption of GRH and close up the gap coming from the factor 2. We shall prove an "almost all" result for this delicate problem, which can be regarded as an analogue, in the higher degree $L$-function case, of the results of Elliott ([6], [7]) and Montgomery \& Vaughan [17] on Dirichlet $L$-functions. It implies that the forms $f$ satisfying (1.10) or (1.11) are rather rare in the sense of having density zero.

In what follows we shall assume $k$ to be any sufficiently large even integer (but the parity will be repeatedly emphasized).

TheOREM 1.1. Fix $m \in\{1,2,3,4\}, \theta_{1}, \theta_{2}>0$ such that $1-2 \theta_{1}-\theta_{2}>0$ and $\theta_{3} \in\left(0, \min \left\{1 / 2 \theta_{1}-1,1\right\}\right]$. Then for $2 \mid k$ and $z \geq\left(\log _{2} k\right)^{1 / \theta_{1}}$, we have

$$
L\left(1, \operatorname{sym}^{m} f\right)=\left\{1+O\left(\frac{1}{z^{\theta_{2}}}+\frac{1}{(\log k)^{\theta_{3}}}\right)\right\} \prod_{p \leq z} \prod_{0 \leq j \leq m}\left(1-\frac{\alpha_{f}(p)^{m-2 j}}{p}\right)^{-1}
$$

for all but $O\left(k e^{-z_{0}^{\theta_{1}}}\right)$ forms $f \in \mathrm{H}_{k}^{*}(1)$, where $z_{0}:=\min \left\{z,(\log k)^{2}\right\}$ and the implied constants depend on $\theta_{1}, \theta_{2}$ and $\theta_{3}$ only. 
Corollary 1.2. Let $\varepsilon>0$ be an arbitrarily small positive number, $m \in$ $\{1,2,3,4\}$ and $2 \mid k$. Then there is a subset $\mathrm{E}_{k}^{*}$ of $\mathrm{H}_{k}^{*}(1)$ such that

$$
\left|\mathrm{E}_{k}^{*}\right| \ll k e^{-(\log k)^{1 / 2-\varepsilon}}
$$

and for each $f \in \mathrm{H}_{k}^{*}(1) \backslash \mathrm{E}_{k}^{*}$, we have

$$
\begin{aligned}
\left\{1+O\left((\log k)^{-\varepsilon}\right)\right\}\left(B_{m}^{-} \log _{2} k\right)^{-A_{m}^{-}} & \leq L\left(1, \operatorname{sym}^{m} f\right) \\
& \leq\left\{1+O\left((\log k)^{-\varepsilon}\right)\right\}\left(B_{m}^{+} \log _{2} k\right)^{A_{m}^{+}}
\end{aligned}
$$

The implied constants depend on $\varepsilon$ only.

REMARKS.

(i) These results can be generalized (with a little extra effort) to $\mathrm{H}_{k}^{*}(N)$, where $N$ is squarefree and $\mathrm{H}_{k}^{*}(N)$ denotes the set of all normalized Hecke primitive eigencuspforms of weight $k$ for the congruence subgroup $\Gamma_{0}(N)$. Our method can also be applied to establish similar results in the level aspect for $N$ squarefree and free of small prime factors.

(ii) We consider the case $1 \leq m \leq 4$ because the required properties of the high symmetric power $L$-functions are only known in these cases. Other higher degree cases will follow along the same lines when the (expected) corresponding properties are established.

Our results above are analogues of Theorem 1 of [17] (see also [7]), where the case $L\left(1, \chi_{d}\right)$ was investigated. However, their methods do not seem to generalize directly to the symmetric power $L$-functions. Following their approach, one can see that the key point of the proof is to study the large sieve type inequality

$$
\sum_{f \in \mathrm{H}_{k}^{*}(1)}\left|\sum_{P<p \leq 2 P} \frac{\lambda_{\mathrm{sym}^{m} f}(p)}{p}\right|^{2 j} .
$$

But then two difficulties come up. First, $\lambda_{\mathrm{sym}^{m} f}(n)$ is not completely multiplicative, and second, the instantaneously available (almost) orthogonality property following from the large sieve result (developed in [5] for the level case and in [14] for the weight) is not adequate. As was indicated by Cogdell \& Michel in [3, Section 1.3], the second difficulty was relatively more serious. In order to get around it, we shall appeal to Petersson's trace formula with the observation $\lambda_{\text {sym }}{ }^{m}(n)=\lambda_{f}\left(n^{m}\right)$ for squarefree $n$. But then the harmonic weight (in the trace formula) needs further treatment as a trivial bound is not suitable for our purpose. To this end, we make use of (see (2.6) below)

$$
1=\frac{k-1}{12} \omega_{f} \sum_{n \leq k^{7 / 2}} \frac{\lambda_{f}\left(n^{2}\right)}{n}+O_{\varepsilon}\left(k^{-1+\varepsilon}\right) \text {, }
$$


where $\omega_{f}$ is the harmonic weight (see (2.5) below). However, only a short initial section of the newly introduced sum is manageable by the Petersson trace formula. The remaining part will be handled using an idea in $[13$, Lemma 6] by means of the large sieve result in [14]. Clearly our result for (1.13) (see the proposition below) is of independent interest and has other applications which will be presented elsewhere.

2. A large sieve type inequality. This section is devoted to establishing a large sieve type inequality, which will be our key tool for the proof of Theorem 1.1. For $2 \mid k, f \in \mathrm{H}_{k}^{*}(1), m \in \mathbb{N}$ and $1 \leq P<Q \leq 2 P$, we consider the sum

$$
T_{\mathrm{sym}^{m} f}(P, Q):=\sum_{P<p \leq Q} \frac{\lambda_{\mathrm{sym}^{m} f}(p)}{p} .
$$

Our aim is to prove the following result, which gives a good control over the tail part of the Dirichlet series representation of $\log L\left(1, \mathrm{sym}^{m} f\right)$ for most forms $f$.

Proposition 2.1. Let $m \in \mathbb{N}$ be fixed. Then

$$
\sum_{f \in \mathrm{H}_{k}^{*}(1)}\left|T_{\operatorname{sym}^{m} f}(P, Q)\right|^{2 j} \ll_{m} k(\log k)^{\theta(m)} e^{2 j \log j} P^{-j}+(j !)^{2} k^{20 / 21}
$$

uniformly for

$$
2 \mid k, \quad j \in \mathbb{N}, \quad 1 \leq P^{j} \leq k^{7 /(6 m+24)}, \quad P<Q \leq 2 P,
$$

where $\theta(m):=(m+1)^{4}+m+7$ and the implied constant depends on $m$ only.

To prove it, we need a couple of preliminary lemmas.

Although the function $\lambda_{\mathrm{sym}^{m} f}(n)$ is not completely multiplicative on $\mathbb{N}$, its restriction to the subset of squarefree integers is, and furthermore

$$
\lambda_{\mathrm{sym}^{m} f}(n)=\prod_{p \mid n} \sum_{0 \leq j \leq m} \alpha_{f}(p)^{m-2 j}=\lambda_{f}\left(n^{m}\right)
$$

for $n$ squarefree, which follows immediately from (1.5)-(1.7). Thus we give an upper estimate for $\left|T_{\mathrm{sym}^{m} f}(P, Q)\right|^{2 j}$ in terms of sums over squarefree integers.

Lemma 2.2. Let $j \in \mathbb{N}, 2 \mid k, m \in \mathbb{N}$ and $1 \leq P<Q \leq 2 P$. For any $f \in \mathrm{H}_{k}^{*}(1)$, we have

$$
\begin{aligned}
& \left|T_{\mathrm{sym}^{m} f}(P, Q)\right|^{2 j} \\
& \quad \ll_{m}(j \log Q)^{(m+1)^{4}} \sum_{n_{2} \leq Q^{j}}^{\natural} \frac{1}{n_{2}^{3 / 2}}\left|\sum_{\substack{P^{j} \\
/ n_{2}<n_{1} \leq Q^{j} / n_{2} \\
\left(n_{1}, n_{2}\right)=1}}^{b} \lambda_{f}\left(n_{1}^{m}\right) \frac{a_{j}\left(n_{1} n_{2}\right)}{n_{1}}\right|^{2},
\end{aligned}
$$


where

$$
a_{j}(n)=a_{j}(n ; P, Q):=\left|\left\{\left(p_{1}, \ldots, p_{j}\right): p_{1} \cdots p_{j}=n, P<p_{i} \leq Q\right\}\right| .
$$

The summations $\sum^{\natural}$ and $\sum^{b}$ run over squarefull $\left({ }^{2}\right)$ and squarefree integers, respectively. The implied constant depends on $m$ only.

Proof. Multiplying out the product $T_{\mathrm{sym}^{m}}(P, Q)^{j}$, we obtain a summation over integers in $\left(P^{j}, Q^{j}\right]$. As every integer $n$ decomposes uniquely into a product of coprime integers $n=n_{1} n_{2}$ with $n_{1}$ squarefree and $n_{2}$ squarefull, it then follows that

$$
\begin{aligned}
& T_{\mathrm{sym}^{m} f}(P, Q)^{j} \\
& \quad=\sum_{n_{2} \leq Q^{j}}^{\natural} \frac{1}{n_{2}} \prod_{p^{\nu} \| n_{2}} \lambda_{\mathrm{sym}^{m} f}(p)^{\nu} \sum_{\substack{P^{j} / n_{2}<n_{1} \leq Q^{j} / n_{2} \\
\left(n_{1}, n_{2}\right)=1}}^{b} \lambda_{\mathrm{sym}^{m} f}\left(n_{1}\right) \frac{a_{j}\left(n_{1} n_{2}\right)}{n_{1}} .
\end{aligned}
$$

Next we remove the products of $\lambda_{\operatorname{sym}^{m} f}(p)$ over squarefull integers by the Cauchy-Schwarz inequality and (1.8):

$$
\begin{aligned}
& \left|T_{\operatorname{sym}^{m} f}(P, Q)\right|^{2 j} \\
& \quad \leq \sum_{n \leq Q^{j}}^{\natural} \frac{(m+1)^{2 \Omega(n)}}{n^{1 / 2}} \sum_{n_{2} \leq Q^{j}}^{\natural} \frac{1}{n_{2}^{3 / 2}}\left|\sum_{\substack{P^{j} \\
/ n_{2}<n_{1} \leq Q^{j} / n_{2} \\
\left(n_{1}, n_{2}\right)=1}}^{b} \lambda_{\mathrm{sym}^{m} f}\left(n_{1}\right) \frac{a_{j}\left(n_{1} n_{2}\right)}{n_{1}}\right|^{2} .
\end{aligned}
$$

Here $\Omega(n)$ denotes the number of prime factors of $n$ counted with multiplicity. Consequently, we get our result with (2.3) and the estimate below obtained by Rankin's trick

$$
\sum_{n \leq x}^{\natural} \frac{(m+1)^{2 \Omega(n)}}{n^{1 / 2}} \leq \prod_{p \leq x}\left(1+\frac{(m+1)^{4}}{p}+O_{m}\left(\frac{1}{p^{3 / 2}}\right)\right) \ll_{m}(\log x)^{(m+1)^{4}}
$$

(see the proofs of Theorems II.1.2 \& II.1.13 in [22] for paradigms).

In view of Lemma 2.2, we invoke naturally the Petersson trace formula to prove our proposition. However, the summation on the left side of (2.1) runs over $f \in \mathrm{H}_{k}^{*}(1)$ without the harmonic weight

$$
\omega_{f}:=\frac{\Gamma(k-1)}{(4 \pi)^{k-1}\|f\|}=\frac{12 \zeta(2)}{(k-1) L\left(1, \operatorname{sym}^{2} f\right)} .
$$

(See $[11, \S 2]$ for the last equality.) We borrow the technique of [13]. The underlying principle is built on approximating the factor $L\left(1, \operatorname{sym}^{2} f\right)$ with a finite Dirichlet series.

$\left({ }^{2}\right)$ An integer $n$ is called squarefull if $p\left|n \Rightarrow p^{2}\right| n$. 
Lemma 2.3. Let $2 \mid k, f \in \mathrm{H}_{k}^{*}(1)$ and $y \geq 1$. For any fixed $\varepsilon>0$, we have

$$
L\left(1, \operatorname{sym}^{2} f\right)=\zeta(2) \sum_{n \leq y} \lambda_{f}\left(n^{2}\right) n^{-1}+O_{\varepsilon}\left(k^{\varepsilon}\left(k^{3 / 4} y^{-1 / 2}+k^{-1}\right)\right) .
$$

The implied constant depends on $\varepsilon$ only.

Proof. For $\sigma>1$, we have

$$
L\left(s, \operatorname{sym}^{2} f\right)=\zeta(2 s) \sum_{n \geq 1} \lambda_{f}\left(n^{2}\right) n^{-s} .
$$

Applying the Perron formula ([22, Corollary II.2.1] with $B(x)=x^{\varepsilon}$ and $\alpha=3$ ), we deduce that

$$
\sum_{n \leq y} \frac{\lambda_{f}\left(n^{2}\right)}{n}=\frac{1}{2 \pi i} \int_{1 / \log y-i k}^{1 / \log y+i k} \frac{L\left(1+s, \operatorname{sym}^{2} f\right)}{\zeta(2+2 s)} \frac{y^{s}}{s} d s+O_{\varepsilon}\left((k y)^{\varepsilon}\left(k^{-1}+y^{-1}\right)\right) .
$$

By moving the segment of integration to $\sigma=-1 / 2+\varepsilon$ and using the convexity bound for $L\left(s, \operatorname{sym}^{2} f\right)$ (see [14, Proposition 3.1]):

$$
L\left(s, \operatorname{sym}^{2} f\right) \ll_{\varepsilon}(k+|\tau|)^{\frac{3}{2} \max \{0,1-\sigma\}+\varepsilon},
$$

it follows that

$$
\sum_{n \leq y} \frac{\lambda_{f}\left(n^{2}\right)}{n}=\frac{L\left(1, \mathrm{sym}^{2} f\right)}{\zeta(2)}+O_{\varepsilon}\left((k y)^{\varepsilon}\left(k^{-1}+k^{3 / 4} y^{-1 / 2}\right)\right)
$$

which is equivalent to the required result.

Taking $y=k^{7 / 2}$ and using the bound $\omega_{f} \ll(\log k) / k(\mathrm{cf}$. [11]), Lemma 2.3 with (2.5) gives

$$
1=\frac{k-1}{12} \omega_{f} \sum_{n \leq y} \frac{\lambda_{f}\left(n^{2}\right)}{n}+O_{\varepsilon}\left(k^{-1+\varepsilon}\right) .
$$

As mentioned in the introduction, the (short enough) initial section of the sum in (2.6) is under control of the Petersson trace formula. For the remaining part, we apply an idea in [13] to deduce that this part is small on average in virtue of the large sieve result developed in [14]. Define

$$
\omega_{f}^{*}(x, y):=\sum_{x<n \leq y} \lambda_{f}\left(n^{2}\right) n^{-1} .
$$

Below we give the analogues of Lemmas 4 and 3 in [13], where the sum $\sum \lambda_{\text {sym }^{2} f}(n) n^{-1}$ is used instead but it seems that our choice will lead to simpler manipulations.

Lemma 2.4. Let $i \geq 1,2 \mid k$ and $f \in \mathrm{H}_{k}^{*}(1)$. Then

$$
\omega_{f}^{*}(x, y)^{i}=\sum_{x^{i}<d \ell \leq y^{i}} \lambda_{f}\left(\ell^{2}\right) \frac{c_{i}(d, \ell)}{d \ell},
$$


where $c_{i}(d, \ell)=0$ unless $d=d^{b} d^{*}$ with $d^{b}$ squarefree and $d^{*}$ squarefull such that $d^{b} \mid \ell$ and $\left(d^{b}, d^{*}\right)=1$. Furthermore,

$$
\left|c_{i}(d, \ell)\right| \leq \tau_{i}(d \ell) \tau_{i-1}(d),
$$

where $\tau_{i}(\cdot)$ is the divisor function defined as in (1.8).

Proof. We proceed by induction on $i$. The case of $i=1$ is trivial since we have $c_{1}(1, \ell)=1$ and $c_{1}(d, \ell)=0$ for $d \geq 2$. Assume that (2.7) holds for $i$ as claimed. Thus by (1.3) we have

$$
\begin{aligned}
\omega_{f}^{*}(x, y)^{i+1} & =\sum_{x<n_{i+1} \leq y} \frac{1}{n_{i+1}} \sum_{x^{i}<d \ell \leq y^{i}} \frac{c_{i}(d, \ell)}{d \ell} \sum_{d_{i} \mid\left(\ell, n_{i+1}\right)^{2}} \lambda_{f}\left(\left(\frac{\ell n_{i+1}}{d_{i}}\right)^{2}\right) \\
& =\sum_{x^{i+1}<d_{0} \ell_{0} \leq y^{i+1}} \frac{\lambda_{f}\left(\ell_{0}^{2}\right)}{d_{0} \ell_{0}} c_{i+1}\left(d_{0}, \ell_{0}\right)
\end{aligned}
$$

with

$$
c_{i+1}\left(d_{0}, \ell_{0}\right)=\sum_{\substack{x<n_{i+1} \leq y \\ \ell n_{i+1}=d_{i} \ell_{0}, d_{0}=d d_{i}}} \sum_{\substack{x_{i}<d \ell \leq y^{i} \\ d_{i} \mid\left(\ell, n_{i+1}\right)^{2}}} c_{i}(d, \ell)
$$

We write uniquely $d_{0}$ as a product $d_{0}^{b} d_{0}^{*}$ of coprime integers with $d_{0}^{b}$ squarefree and $d_{0}^{*}$ squarefull. We claim that

$$
c_{i+1}\left(d_{0}, \ell_{0}\right) \neq 0 \Rightarrow d_{0}^{b} \mid \ell_{0} .
$$

Let $d_{0}^{b}=d^{\prime} d_{i}^{\prime}$ with $d^{\prime} \| d$ and $d_{i}^{\prime} \| d_{i}\left({ }^{3}\right)$. Then, $\left(d^{\prime}, d_{i}\right)=\left(d_{i}^{\prime}, d\right)=1$ as $d_{0}^{b} \| d d_{i}$ and $d_{0}^{b}$ is squarefree. Since $d_{i} \mid\left(\ell, n_{i+1}\right)^{2}$ and $\ell n_{i+1}=d_{i} \ell_{0}$, we have $d_{i}^{\prime} \mid \ell_{0}$ (by noting $\left.d_{i}^{\prime} \| d_{i}\right)$. On the other hand, by the induction hypothesis we see that $c_{i}(d, \ell) \neq 0$ implies $d^{\prime} \mid \ell$, thus $d^{\prime} \mid \ell_{0}$ for $\left(d^{\prime}, d_{i}\right)=1$. This implies $d_{0}^{b} \mid \ell_{0}$ as $d_{0}^{b}=d^{\prime} d_{i}^{\prime}$ is squarefree.

It remains to verify (2.8), which is an immediate consequence of the formula

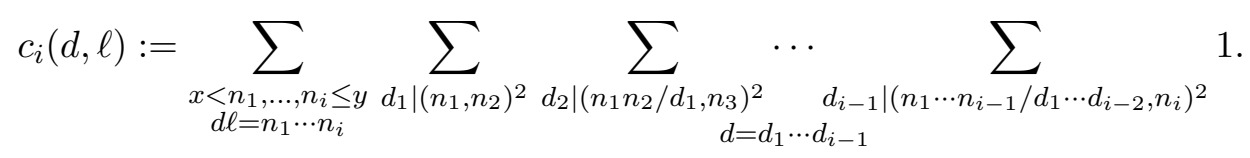

This completes the proof of Lemma 2.4.

Lemma 2.5. For any $A>0, \varepsilon>0$ and integer $i \geq 1$, we have

$$
\sum_{f \in \mathrm{H}_{k}^{*}(1)} \omega_{f}^{*}(x, y)^{2 i} \ll_{A, \varepsilon, i} k^{\varepsilon}
$$

uniformly for $2 \mid k$ and $k^{5} \leq x^{i}<y^{i} \leq k^{A}$.

$\left({ }^{3}\right)$ The notation $d \| n$ means that $v_{p}(d)=v_{p}(n)$ for all $p \mid d$, where $v_{p}(n)$ is the exponent of $p$ in the canonical factorization of $n$. 
Proof. The main ingredients of the proof are Lemma 2.4 and the following large sieve type inequality: Suppose $a(n) \ll_{\varepsilon} n^{-1+\varepsilon}$ for any $\varepsilon>0$. Then

$$
\sum_{f \in \mathrm{H}_{k}^{*}(1)}\left|\sum_{L<\ell \leq 2 L} a(\ell) \lambda_{f}\left(\ell^{2}\right)\right|^{2} \ll_{\varepsilon}(k L)^{\varepsilon}\left(1+k^{5 / 2} L^{-1 / 2}\right)
$$

uniformly for $2 \mid k$ and $L \geq 1$.

The inequality (2.10) is a consequence of the relation

$$
\lambda_{f}\left(\ell^{2}\right)=\sum_{d n^{2}=\ell} \lambda_{\mathrm{sym}^{2} f}(d) \mu(n),
$$

where $\mu(n)$ is the Möbius function, and the large sieve inequality in [14, Proposition 4.1] with $m=2$ : For any $\varepsilon>0$ we have

$$
\sum_{f \in \mathrm{H}_{k}^{*}(1)}\left|\sum_{\ell \leq L} b_{\ell} \lambda_{\mathrm{sym}^{2} f}(\ell)\right|^{2} \ll_{\varepsilon} k^{\varepsilon}\left(L+k^{5 / 2} L^{1 / 2+\varepsilon}\right) \sum_{\ell \leq L}\left|b_{\ell}\right|^{2}
$$

uniformly for $2 \mid k, L \geq 1$ and $\left\{b_{\ell}\right\}_{1 \leq \ell \leq L} \subset \mathbb{C}$.

From (2.11), we write the inner sum in (2.10) as

$$
\sum_{L<\ell \leq 2 L} a(\ell) \lambda_{f}\left(\ell^{2}\right)=\sum_{d \leq 2 L} \lambda_{\mathrm{sym}^{2} f}(d) \sum_{\sqrt{L / d}<n \leq \sqrt{2 L / d}} \mu(n) a\left(d n^{2}\right)
$$

and apply the large sieve inequality to the right side. Then (2.10) follows because the condition $a(n) \ll n^{-1+\varepsilon}$ yields

$$
\sum_{d \leq 2 L}\left|\sum_{\sqrt{L / d}<n \leq \sqrt{2 L / d}}\right| a\left(d n^{2}\right)||^{2} \ll L^{-1+\varepsilon}
$$

Now we prove (2.9). Firstly, we divide the sum in (2.7) dyadically:

$$
\omega_{f}^{*}(x, y)^{i}=\sum_{j \leq\left(\log y^{i}\right) / \log 2} \sum_{x^{i} / 2^{j+1}<\ell \leq y^{i} / 2^{j}} \lambda_{f}\left(\ell^{2}\right) \frac{c_{j}(\ell)}{\ell},
$$

where

$$
c_{j}(\ell):=\sum_{\substack{2^{j}<d \leq 2^{j+1} \\ x^{i} / \ell<d \leq y^{i} / \ell}} \frac{c_{i}(d, \ell)}{d} .
$$

Then, by the Cauchy-Schwarz inequality, we obtain

$$
\begin{aligned}
& \sum_{f \in \mathrm{H}_{k}^{*}(1)} \omega_{f}^{*}(x, y)^{2 i} \\
& \ll_{A}(\log k) \sum_{j \leq\left(\log y^{i}\right) / \log 2} \sum_{f \in \mathrm{H}_{k}^{*}(1)}\left|\sum_{x^{i} / 2^{j+1}<\ell \leq y^{i} / 2^{j}} \lambda_{f}\left(\ell^{2}\right) \frac{c_{j}(\ell)}{\ell}\right|^{2} .
\end{aligned}
$$


From $(2.8)$ and $\tau_{i}(d \ell) \leq \tau_{i}(d) \tau_{i}(\ell)$, we have

$$
c_{j}(\ell) \leq \tau_{i}(\ell)^{3} \sum_{d \mid \ell} \frac{1}{d} \sum_{2^{j} / d<d^{*} \leq 2^{j+1} / d}^{\natural} \frac{\tau_{i}\left(d^{*}\right)^{2}}{d^{*}} .
$$

By the Rankin trick again, it is easy to see that

$$
\sum_{d \leq t}^{\natural} \frac{\tau_{i}(d)^{2}}{\sqrt{d}} \ll(\log t)^{\theta_{0}(i)}
$$

with $\theta_{0}(i):=((i+1) i / 2)^{2}$, and hence

$$
c_{j}(\ell) \ll \tau_{i}(\ell)^{3} \tau(\ell) 2^{-j / 2}\left(\log 2^{j}\right)^{\theta_{0}(i)} .
$$

From (2.12) and (2.10) with $a(\ell)=2^{j / 2}\left(\log 2^{j}\right)^{-\theta_{0}(i)} c_{j}(\ell) / \ell$, we infer that

$$
\sum_{f \in \mathrm{H}_{k}^{*}(1)} \omega_{f}^{*}(x, y)^{2 i}
$$

$$
\begin{aligned}
& \ll_{A, \varepsilon, i} k^{\varepsilon} \sum_{j \ll \log k} \frac{1}{2^{j}} \sum_{f \in \mathrm{H}_{k}^{*}(1)}\left|\sum_{x^{i} / 2^{j+1}<\ell \leq y^{i} / 2^{j}} \frac{2^{j / 2}\left(\log 2^{j}\right)^{-\theta_{0}(i)} c_{j}(\ell)}{\ell} \lambda_{f}\left(\ell^{2}\right)\right|^{2} \\
& \ll_{A, \varepsilon, i} k^{\varepsilon} \sum_{j \ll \log k} 2^{-j}\left\{1+k^{5 / 2}\left(x^{i} 2^{-j-1}\right)^{-1 / 2}\right\} \ll_{A, \varepsilon, i} k^{\varepsilon}
\end{aligned}
$$

for $k^{5} \leq x^{i} \leq y^{i} \leq k^{A}$.

Now we are ready to prove Proposition 2.1.

Proof of Proposition 2.1. By Lemma 2.2 and (2.6), we deduce that

$$
\begin{aligned}
\sum_{f \in \mathrm{H}_{k}^{*}(1)}\left|T_{\mathrm{sym}^{m} f}(P, Q)\right|^{2 j} & \\
& \ll_{m}(\log k)^{(m+1)^{4}}\left(k \sum_{n_{2} \leq Q^{j}}^{\natural} \frac{\left|M\left(n_{2}\right)\right|}{n_{2}^{3 / 2}}+O(R)\right),
\end{aligned}
$$

where

$$
M\left(n_{2}\right):=\sum_{f \in \mathrm{H}_{k}^{*}(1)} \omega_{f} \sum_{n \leq y} \frac{\lambda_{f}\left(n^{2}\right)}{n}\left|\sum_{\substack{P^{j} \\ p_{2}<n_{1} \leq Q^{j} / n_{2} \\\left(n_{1}, n_{2}\right)=1}}^{b} \lambda_{f}\left(n_{1}^{m}\right) \frac{a_{j}\left(n_{1} n_{2}\right)}{n_{1}}\right|^{2}
$$

with $y=k^{7 / 2}$, and

$$
R:=k^{-1+\varepsilon} \sum_{n_{2} \leq Q^{j}}^{\natural} \frac{1}{n_{2}^{3 / 2}} \sum_{f \in \mathrm{H}_{k}^{*}(1)}\left|\sum_{\substack{P^{j} / n_{2}<n_{1} \leq Q^{j} / n_{2} \\\left(n_{1}, n_{2}\right)=1}}^{b} \lambda_{f}\left(n_{1}^{m}\right) \frac{a_{j}\left(n_{1} n_{2}\right)}{n_{1}}\right|^{2}
$$




$$
\ll j !^{2} k^{\varepsilon} \sum_{n_{2} \leq Q^{j}}^{\natural} \frac{1}{n_{2}^{3 / 2}}\left(\sum_{P^{j} / n_{2}<n \leq Q^{j} / n_{2}} \frac{\tau\left(n^{m}\right)}{n}\right)^{2} \ll j !^{2} k^{\varepsilon}
$$

by the Deligne inequality, (1.1) and the trivial estimate for (2.4),

$$
a_{j}(n) \leq j !
$$

The remaining task is to estimate $M\left(n_{2}\right)$. We square out the innermost sum in $M\left(n_{2}\right)$ and explore the cancellation through the Petersson trace formula. But this approach is only effective for small $n$, hence we split $M\left(n_{2}\right)$ into two parts,

$$
M\left(n_{2}\right)=S_{x}+S_{x, y}
$$

according to $n \leq x$ and $x<n \leq y$ respectively where $x=k^{1 / 2}$. The second term $S_{x, y}$ is handled by Lemma 2.5, as follows.

From the estimate $\omega_{f} \ll(\log k) / k$, the Deligne inequality and $(2.15)$, we have

$$
\begin{aligned}
S_{x, y} & \ll k^{-1}(\log k) \sum_{f \in \mathrm{H}_{k}^{*}(1)}\left|\omega_{f}^{*}(x, y)\right|\left(j ! \sum_{n \leq Q^{j}} \tau\left(n^{m}\right) n^{-1}\right)^{2} \\
& \ll(j !)^{2} k^{-1}(\log k)^{2 m+3} \sum_{f \in \mathrm{H}_{k}^{*}(1)}\left|\omega_{f}^{*}(x, y)\right| .
\end{aligned}
$$

Applying Hölder's inequality and Lemma 2.5 with $i=10$, we deduce that

$$
S_{x, y} \ll(j !)^{2} k^{-1+\varepsilon}\left(\sum_{f \in \mathrm{H}_{k}^{*}(1)} 1\right)^{19 / 20}\left(\sum_{f \in \mathrm{H}_{k}^{*}(1)} \omega_{f}^{*}(x, y)^{20}\right)^{1 / 20} \ll(j !)^{2} k^{-1 / 20+\varepsilon} \text {. }
$$

Now we treat $S_{x}$ by the Petersson trace formula (see [12, Corollary 2.10])

$$
\sum_{f \in \mathrm{H}_{k}^{*}(1)} \omega_{f} \lambda_{f}(a) \lambda_{f}(b)=\delta(a, b)+O\left(k^{-5 / 6}(a b)^{1 / 4} \tau_{3}((a, b)) \log (2 a b)\right),
$$

where $\delta(a, b)$ is the Kronecker delta and the implied constant is absolute. Squaring out and using (1.3) and (2.15), we obtain

$$
\begin{aligned}
S_{x} \leq & (j !)^{2} \sum_{n \leq x} \frac{1}{n} \sum_{P^{j}}^{b} \sum_{n_{2}<n_{1}, n_{1}^{\prime} \leq Q^{j} / n_{2}}^{b} \frac{1}{n_{1} n_{1}^{\prime}} \\
& \times \sum_{d \mid\left(n_{1}, n_{1}^{\prime}\right)^{m}}\left|\sum_{f \in \mathrm{H}_{k}^{*}(1)} \omega_{f} \lambda_{f}\left(n^{2}\right) \lambda_{f}\left(\frac{\left(n_{1} n_{1}^{\prime}\right)^{m}}{d^{2}}\right)\right| .
\end{aligned}
$$

Let us write $n_{1}=d \ell$ and $n_{1}^{\prime}=d \ell^{\prime}$ where $d=\left(n_{1}, n_{1}^{\prime}\right)$. Then $d, \ell$ and $\ell^{\prime}$ are squarefree and pairwise coprime for squarefree $n_{1}$ and $n_{1}^{\prime}$. Therefore, 


$$
\begin{aligned}
S_{x} \leq & (j !)^{2} \sum_{\substack{P^{j} / n_{2}<d \ell, d \ell^{\prime} \leq Q^{j} / n_{2} \\
\left(\ell, \ell^{\prime}\right)=1}}^{b} \frac{1}{d^{2} \ell \ell^{\prime}} \\
& \times \sum_{d_{1} \mid d^{m}} \sum_{n \leq x} \frac{1}{n}\left|\sum_{f \in \mathrm{H}_{k}^{*}(1)} \omega_{f} \lambda_{f}\left(n^{2}\right) \lambda_{f}\left(\frac{\left(d^{2} \ell \ell^{\prime}\right)^{m}}{d_{1}^{2}}\right)\right|
\end{aligned}
$$

The Petersson trace formula shows that the sum over $f \in \mathrm{H}_{k}^{*}(1)$ equals

$$
\delta\left(n^{2},\left(\ell \ell^{\prime}\right)^{m}\left(d^{m} / d_{1}\right)^{2}\right)+O\left(\frac{\left(d^{2} \ell \ell^{\prime}\right)^{m / 4} n^{1 / 2}}{d_{1}^{1 / 2} k^{5 / 6}} \tau_{3}\left(n^{2}\right) \log k\right) .
$$

Clearly for $d_{1} \mid d^{m}$ and squarefree integers $\ell$ and $\ell^{\prime}$ with $\left(\ell, \ell^{\prime}\right)=1$, we have

$$
n^{2}=\left(\ell \ell^{\prime}\right)^{m}\left(d^{m} / d_{1}\right)^{2} \Rightarrow \ell \ell^{\prime}\left(d^{m} / d_{1}\right) \mid n \text {. }
$$

Thus after summing over $n$, the $\delta$-symbol contributes

$$
\sum_{n \leq x} \frac{1}{n} \delta\left(n^{2},\left(\ell \ell^{\prime}\right)^{m}\left(d^{m} / d_{1}\right)^{2}\right) \ll \frac{1}{\ell \ell^{\prime}} \frac{d_{1}}{d^{m}} \sum_{n \leq x / \ell \ell^{\prime}\left(d^{m} / d_{1}\right)} \frac{1}{n} \ll \frac{\log k}{\ell \ell^{\prime}},
$$

while the $O$-term produces a term trivially bounded by

$$
\frac{\log k}{\ell \ell^{\prime}} \frac{Q^{j(m / 2+2)}}{k^{5 / 6}} \sum_{n \leq x} \frac{\tau_{3}\left(n^{2}\right)}{\sqrt{n}} \ll(\log k)^{6} \ell \ell^{\prime}
$$

in view of our choices of $x, j$ and $Q$.

Inserting these estimates into (2.17), it follows that

$$
\begin{aligned}
S_{x} & \ll(j !)^{2}(\log k)^{6} \sum_{P^{j} / n_{2}<d \ell, d \ell^{\prime} \leq Q^{j} / n_{2}}^{b} \sum_{d \leq \ell^{j} / n_{2}}^{b} \frac{\tau\left(d^{m}\right)}{d^{2}}\left(\sum_{P^{j} / d n_{2}<\ell \leq Q^{j} / d n_{2}}^{b} \frac{1}{\ell^{2}}\right)^{2} \\
& \ll(j !)^{2}(\log k)^{6} \sum_{d \leq \ell^{\prime}}^{b} \frac{\tau\left(d^{m}\right)}{\sum_{d \leq Q^{j} / n_{2}}^{b}} \tau\left(d^{m}\right) .
\end{aligned}
$$

Together with the estimates of $S_{x, y}$ and (2.17), we get an upper bound for $M\left(n_{2}\right)$ :

$$
M\left(n_{2}\right) \ll(j !)^{2}(\log k)^{6} \frac{n_{2}^{2}}{P^{2 j}} \sum_{d \leq Q^{j} / n_{2}}^{b} \tau\left(d^{m}\right)+(j !)^{2} k^{-1 / 20+\varepsilon} .
$$

In view of (2.13), we need to evaluate the following sum over squarefull integers:

$$
\sum_{n \leq Q^{j}}^{\natural} n^{1 / 2} \sum_{d \leq Q^{j} / n}^{b} \tau\left(d^{m}\right) \ll Q^{j}(\log k)^{m} \sum_{n \leq Q^{j}}^{\natural} n^{-1 / 2} \ll Q^{j}(\log k)^{m+1}
$$


as there are $O(\sqrt{t})$ squarefull integers less than $t$ and

$$
\sum_{d \leq t} \tau\left(d^{m}\right) \ll t(\log t)^{m}
$$

Together with (2.13) and (2.14), we conclude that

$$
\sum_{f \in \mathrm{H}_{k}^{*}(1)}\left|T_{\mathrm{sym}^{m} f}(P, Q)\right|^{2 j} \ll k(\log k)^{(m+1)^{4}+m+7}(j !)^{2} Q^{j} P^{-2 j}+(j !)^{2} k^{19 / 20+\varepsilon},
$$

which gives our desired result, by Stirling's formula and $Q \leq 2 P$.

3. Proof of Theorem 1.1. Let $m \in \mathbb{N}, 2 \mid k$ and $f \in \mathrm{H}_{k}^{*}(1)$. We have

$$
\log L\left(s, \operatorname{sym}^{m} f\right)=\sum_{n=1}^{\infty} \frac{\Lambda_{\mathrm{sym}^{m} f}(n)}{n^{s} \log n} \quad(\sigma>1)
$$

where

$$
\begin{aligned}
& \Lambda_{\mathrm{sym}^{m} f}(n) \\
& \quad= \begin{cases}{\left[\alpha_{f}(p)^{m \nu}+\alpha_{f}(p)^{(m-2) \nu}+\cdots+\alpha_{f}(p)^{-m \nu}\right] \log p} & \text { if } n=p^{\nu} \\
0 & \text { otherwise. }\end{cases}
\end{aligned}
$$

Apparently $\left|\Lambda_{\text {sym }^{m} f}(n)\right| \leq(m+1) \log n$ for $n \geq 1$. To prove our theorem, we shall show that for almost all $f, \log L\left(1, \operatorname{sym}^{m} f\right)$ is well approximated by a short partial sum over primes. Actually, $\log L\left(1, \operatorname{sym}^{m} f\right)$ has a good approximation by a partial sum of moderate length when $L\left(s, \operatorname{sym}^{m} f\right)$ has a bigger zero-free region, which is available for most $f \in \mathrm{H}_{k}^{*}(1)$.

As in [14], for each $\eta \in(0,1 / 100]$, we define

$$
\mathrm{H}_{k, \mathrm{sym}^{m}}^{+}(1 ; \eta):=\left\{f \in \mathrm{H}_{k}^{*}(1): L\left(s, \operatorname{sym}^{m} f\right) \neq 0 \text { for } s \in \mathcal{S}\right\},
$$

where $\mathcal{S}:=\left\{s: \sigma \geq 1-\eta,|\tau| \leq 100 k^{\eta}\right\} \cup\{s: \sigma \geq 1\}$, and

$$
\mathrm{H}_{k, \mathrm{sym}^{m}}^{-}(1 ; \eta):=\mathrm{H}_{k}^{*}(1) \backslash \mathrm{H}_{k, \mathrm{sym}^{m}}^{+}(1 ; \eta) .
$$

According to (1.11) of [14], we have

$$
\left|\mathrm{H}_{k, \mathrm{sym}^{m}}^{-}(1 ; \eta)\right| \ll_{\eta} k^{31 \eta}
$$

For $f \in \mathrm{H}_{k, \mathrm{sym}^{m}}^{+}(1 ; \eta)$, we have the following result.

Lemma 3.1. Fix $\eta \in(0,1 / 100]$ and $\delta_{0} \in(0,1]$ and let $m \in\{1,2,3,4\}$. Let $2 \mid k$ and $x=\exp \left\{[(\log k) / 7(m+4)]^{\delta_{0}}\right\}$. Then for any $f \in \mathrm{H}_{k, \mathrm{sym}^{m}}^{+}(1 ; \eta)$, we have

$$
\log L\left(1, \operatorname{sym}^{m} f\right)=\sum_{p \leq x} \sum_{0 \leq j \leq m} \log \left(1-\frac{\alpha_{f}(p)^{m-2 j}}{p}\right)^{-1}+O\left(\frac{1}{(\log k)^{\delta_{0}}}\right) .
$$

The implied constant depends on $\delta, \eta$ and $m$ only. 
Proof. Let $f \in \mathrm{H}_{k}^{*}(1), T \geq 1$ and $x \geq 1$. By the Perron formula ([22, Corollary II.2.1] with $B(x)=1$ and $\alpha=1$ ), we have

$$
\begin{aligned}
\sum_{2 \leq n \leq x} \frac{\Lambda_{\operatorname{sym}^{m} f}(n)}{n \log n}= & \frac{1}{2 \pi i} \int_{1 / \log x-i T}^{1 / \log x+i T} \log L\left(s+1, \operatorname{sym}^{m} f\right) \frac{x^{s}}{s} d s \\
& +O\left(\frac{\log (T x)}{T}+\frac{1}{x}\right) .
\end{aligned}
$$

Once $f \in \mathrm{H}_{k, \mathrm{sym}^{m}}^{+}(1 ; \eta)$, we have the upper estimate

$$
\log L\left(s, \operatorname{sym}^{m} f\right) \ll_{\eta} \log k
$$

uniformly for $\sigma \geq 1-\frac{1}{4} \eta$ and $|\tau| \leq(\log k)^{4 / \eta}$. This is a particular case of Proposition 3.5 of [14] (with $\alpha=\frac{1}{4} \eta$ ).

Now for $f \in \mathrm{H}_{k, \mathrm{sym}^{m}}^{+}(1 ; \eta)$, we move the line of integration to $\sigma=-\frac{1}{4} \eta$ and estimate $\log L\left(s+1, \operatorname{sym}^{m} f\right)$ by (3.6) over the contour. We see that

$$
\begin{aligned}
\sum_{2 \leq n \leq x} \frac{\Lambda_{\operatorname{sym}^{m} f}(n)}{n \log n} & \\
= & \log L\left(1, \operatorname{sym}^{m} f\right)+O\left(\frac{\log (k T x)}{T}+\frac{(\log k)(\log T)}{x^{\eta / 4}}\right) \\
& =\log L\left(1, \operatorname{sym}^{m} f\right)+O\left(\frac{1}{(\log k)^{4 / \eta-1}}\right)
\end{aligned}
$$

by taking the parameters $T=(\log k)^{4 / \eta}$ and $x=\exp \left\{[(\log k) / 7(m+4)]^{\delta_{0}}\right\}$.

On the other hand, we have

$$
\begin{aligned}
\sum_{2 \leq n \leq x} \frac{\Lambda_{\mathrm{sym}^{m} f}(n)}{n \log n} & =\sum_{p \leq x} \sum_{\nu \leq(\log x) / \log p} \frac{\Lambda_{\mathrm{sym}^{m} f}\left(p^{\nu}\right)}{p^{\nu} \log p^{\nu}} \\
& =\sum_{p \leq x} \sum_{0 \leq j \leq m} \sum_{\nu \leq(\log x) / \log p} \frac{\alpha_{f}(p)^{(m-2 j) \nu}}{\nu p^{\nu}} \\
& =\sum_{p \leq x} \sum_{0 \leq j \leq m}\left\{\log \left(1-\frac{\alpha_{f}(p)^{m-2 j}}{p}\right)^{-1}+O\left(\frac{1}{x}\right)\right\}
\end{aligned}
$$

Combining (3.7) and (3.8), we get the required result.

The size of $x$ given in Lemma 3.1, even though being quite small, is still insufficient for our purpose. Making use of the proposition to remove the "exceptional forms", we are able to further reduce its size in the next two lemmas.

Lemma 3.2. Fix $m \in \mathbb{N}$ and $\delta_{1}, \delta_{2}>0$ such that $\delta_{1}-\delta_{2}-2>0$. Suppose that

$$
2 \mid k \quad \text { and } \quad(\log k)^{\delta_{1}} \leq P \leq Q \leq 2 P \leq k^{14 / 15(m+4)} .
$$


Then

$$
\left|T_{\text {sym }^{m} f}(P, Q)\right| \leq \frac{1}{(\log k)^{\delta_{2}}}
$$

for all but $O_{\delta_{1}, \delta_{2}, m}\left(k^{1-\theta_{0}}\right)$ forms $f \in \mathrm{H}_{k}^{*}(1)$, where

$$
\theta_{0}:=\left(\delta_{1}-\delta_{2}-2\right) / 10(m+4) \delta_{1}>0 \text {. }
$$

Proof. Define

$$
\mathrm{E}_{m}^{1}(P, Q):=\left\{f \in \mathrm{H}_{k}^{*}(1):(3.10) \text { fails }\right\} .
$$

We shall use Proposition 2.1 with the choices

$$
j=\left[c^{\prime} \frac{\log k}{\log P}\right]+1, \quad c^{\prime}:=\frac{1}{5(m+4)}
$$

to count $\left|\mathrm{E}_{m}^{1}(P, Q)\right|$. Plainly we have

$$
k^{1 /(3 m+12)} \leq P^{j}<(2 P)^{j} \leq k^{7 /(6 m+24)}
$$

by (3.9), whence the proposition is applicable. It follows that

$$
\left|\mathrm{E}_{m}^{1}(P, Q)\right| \ll k\left((\log k)^{\theta(m)} e^{2 j \log j} P^{-j}+e^{2 j \log j} k^{-1 / 21}\right)(\log k)^{2 \delta_{2} j} .
$$

On the other hand, the lower bound for $P$ in (3.9) yields

$-j \log P+j\left(2 \log j+\delta_{2} \log _{2} k\right)+\theta(m) \log _{2} k$

$$
\begin{aligned}
& \leq-c^{\prime} \log k+\left(c^{\prime}(\log k) / \log P+1\right)\left(2+\delta_{2}\right) \log _{2} k+\theta(m) \log _{2} k \\
& \leq-c^{\prime}\left\{\left(\delta_{1}-\delta_{2}-2\right) / \delta_{1}\right\} \log k+\left(\theta(m)+2+\delta_{2}\right) \log _{2} k \\
& \leq-\frac{1}{2} c^{\prime}\left\{\left(\delta_{1}-\delta_{2}-2\right) / \delta_{1}\right\} \log k
\end{aligned}
$$

and

$$
\begin{aligned}
-\frac{1}{21} \log k+j(2 \log j+ & \left.\delta_{2} \log _{2} k\right) \\
& \leq-\frac{1}{21} \log k+\left(c^{\prime}(\log k) / \log P+1\right)\left(2+\delta_{2}\right) \log _{2} k \\
& \leq-\left(\frac{1}{21}-c^{\prime}\left(2+\delta_{2}\right) / \delta_{1}\right) \log k+\left(2+\delta_{2}\right) \log _{2} k \\
& \leq-\frac{1}{2}\left(\frac{1}{21}-c^{\prime}\left(2+\delta_{2}\right) / \delta_{1}\right) \log k .
\end{aligned}
$$

Inserting these two estimates into (3.12) and noticing $\frac{1}{21}-c^{\prime}\left(2+\delta_{2}\right) / \delta_{1} \geq$ $c^{\prime}\left(\delta_{1}-\delta_{2}-2\right) / \delta_{1}$, we get the desired result. This completes the proof.

Lemma 3.3. Fix $m \in \mathbb{N}$ and $\delta_{3}, \delta_{4}>0$ such that $1-2 \delta_{3}-\delta_{4}>0$. Suppose that

$$
2 \mid k \quad \text { and } \quad\left(\log _{2} k\right)^{1 / \delta_{3}} \leq P \leq Q \leq 2 P \leq(c \log k)^{1 / \delta_{3}},
$$
where $c=\left(1-2 \delta_{3}-\delta_{4}\right) / 24(m+4)(\theta(m)+2)>0$. Then

$$
\left|T_{\text {sym }^{m} f}(P, Q)\right| \leq P^{-\delta_{4}}
$$

for all but $O_{\delta_{3}, \delta_{4}, m}\left(k e^{-(\theta(m)+2) P^{\delta_{3}}}\right)$ forms $f \in \mathrm{H}_{k}^{*}(1)$. 
Proof. The argument is similar to Lemma 3.2. Define

$$
\mathrm{E}_{m}^{2}(P, Q):=\left\{f \in \mathrm{H}_{k}^{*}(1):(3.14) \text { fails }\right\} .
$$

This time we apply Proposition 2.1 with another choice of parameters:

$$
j=\left[c^{\prime} \frac{P^{\delta_{3}}}{\log P}\right]+1, \quad c^{\prime}:=\frac{2 \theta(m)+4}{1-2 \delta_{3}-\delta_{4}} .
$$

By (3.13), it is easy to verify that $e^{c^{\prime} P^{\delta_{3}}} \leq P^{j} \leq k^{2 c c^{\prime}}=k^{1 / 6(m+4)}<$ $k^{7 /(6 m+24)}$. Thus we deduce by the proposition that

$$
\left|\mathrm{E}_{m}^{2}(P, Q)\right| \ll k\left((\log k)^{\theta(m)} e^{2 j \log j} P^{-j}+e^{2 j \log j} k^{-1 / 21}\right) P^{2 \delta_{4} j} .
$$

Now, in view of our choices of $c^{\prime}$ and $c$, we have

$$
\begin{aligned}
-(1- & \left.\delta_{4}\right) j \log P+2 j \log j+\theta(m) \log _{2} k \\
& \leq-\left(1-\delta_{4}\right) c^{\prime} P^{\delta_{3}}+2\left(c^{\prime} P^{\delta_{3}} / \log P+1\right) \delta_{3} \log P+\theta(m) \log _{2} k \\
& \leq-c^{\prime}\left(1-2 \delta_{3}-\delta_{4}\right) P^{\delta_{3}}+(\theta(m)+2) \log _{2} k \\
& \leq-\frac{1}{2} c^{\prime}\left(1-2 \delta_{3}-\delta_{4}\right) P^{\delta_{3}}
\end{aligned}
$$

by the lower bound for $P$ in (3.13), and

$$
\begin{aligned}
-\frac{1}{21} \log k+j(2 \log j & \left.+\delta_{4} \log P\right) \\
& \leq-\frac{1}{21} \log k+\left(c^{\prime} P^{\delta_{3}} / \log P+1\right)\left(2 \delta_{3}+\delta_{4}\right) \log P \\
& \leq-\frac{1}{21} \log k+2 c^{\prime}\left(2 \delta_{3}+\delta_{4}\right) P^{\delta_{3}} \\
& \leq-\left(\frac{1}{21 c}-2 c^{\prime}\left(2 \delta_{3}+\delta_{4}\right)\right) P^{\delta_{3}} \\
& \leq-\frac{1}{2} c^{\prime}\left(1-2 \delta_{3}-\delta_{4}\right) P^{\delta_{3}}
\end{aligned}
$$

by the upper bound in (3.13). We get the required result by these two estimates and (3.16). This completes the proof.

Now we finish the proof of Theorem 1.1. Fix $\eta \in(0,1 / 100]$ and $\delta_{0} \in(0,1]$ and let $m \in\{1,2,3,4\}$. Take $\delta_{i}(1 \leq i \leq 4)$ such that

$$
1 / \theta_{1}>\delta_{1}>2 /\left(1-\theta_{2}\right), \quad \delta_{2}=2 \delta_{0}=2 \theta_{3}, \quad \delta_{3}=\theta_{1}, \quad \delta_{4}=\theta_{2} .
$$

It is easy to verify that $\delta_{1}$ and $\delta_{3}$ fulfill the conditions in Lemmas 2.4 and 2.5 respectively, and $1 / \delta_{3}>\delta_{1}$. Define

$$
x=\exp \left\{[(\log k) / 7(m+4)]^{\delta_{0}}\right\}, \quad y_{1}:=(\log k)^{\delta_{1}}, \quad y_{2}:=\left(\log _{2} k\right)^{1 / \delta_{3}} .
$$

Then we consider the following three cases according to the size of $z$.

$1^{\circ}$ The case $z \geq x$. The required formula follows immediately from Lemma 3.1 with a better upper bound $O\left(k^{31 \eta}\right)$ for the exceptional set in view of (3.5). 
$2^{\mathrm{o}}$ The case $y_{1} \leq z<x$. By Lemma 3.1 with $x=\exp \left\{[(\log k) / 7(m+4)]^{\delta_{0}}\right\}$, we can write

$$
\begin{aligned}
& \log L\left(1, \operatorname{sym}^{m} f\right) \\
= & \sum_{p \leq z} \sum_{0 \leq j \leq m} \log \left(1-\frac{\alpha_{f}(p)^{m-2 j}}{p}\right)^{-1}+O\left(\frac{1}{(\log k)^{\delta_{0}}}\right)+R_{1}\left(\operatorname{sym}^{m} f\right)
\end{aligned}
$$

for any $f \in \mathrm{H}_{k, \mathrm{sym}^{m}}^{+}(1 ; \eta)$, where

$$
R_{1}\left(\operatorname{sym}^{m} f\right):=-\sum_{z<p \leq x} \sum_{0 \leq j \leq m} \log \left(1-\frac{\alpha_{f}(p)^{m-2 j}}{p}\right) .
$$

This case will be done if we show that $R_{1}\left(\operatorname{sym}^{m} f\right)$ is negligible apart from a small exceptional set of $f$. Clearly,

$$
\begin{aligned}
R_{1}\left(\operatorname{sym}^{m} f\right) & =\sum_{z<p \leq x}\left\{\frac{\lambda_{\operatorname{sym}^{m} f}(p)}{p}+O_{m}\left(\frac{1}{p^{2}}\right)\right\} \\
& =\sum_{z<p \leq x} \frac{\lambda_{\operatorname{sym}^{m} f}(p)}{p}+O\left(\frac{1}{z}\right) .
\end{aligned}
$$

Define

$$
P_{i}:=2^{i-1} z, \quad Q_{i}:=\min \left\{2^{i} z, x\right\}, \quad \mathrm{E}_{m}^{1}:=\mathrm{H}_{k, \mathrm{sym}^{m}}^{-}(1 ; \eta) \cup \bigcup_{i \ll \log x} \mathrm{E}_{m}^{1}\left(P_{i}, Q_{i}\right),
$$

where $\mathrm{E}_{m}^{1}\left(P_{i}, Q_{i}\right)$ is defined as in (3.11). According to Lemma 3.2, we have

$$
\left|\mathrm{E}_{m}^{1}\right| \ll k^{31 \eta}+\sum_{i \ll \log x}\left|\mathrm{E}_{m}^{1}\left(P_{i}, Q_{i}\right)\right| \ll(\log k)^{\delta_{0}} k^{1-\theta_{0}}
$$

and for $f \notin \mathrm{E}_{m}^{1}$,

$$
R_{1}\left(\operatorname{sym}^{m} f\right) \ll \sum_{i \ll \log x}\left|T_{\operatorname{sym}^{m} f}\left(P_{i}, Q_{i}\right)\right|+\frac{1}{z} \ll \frac{1}{(\log k)^{\delta_{2}-\delta_{0}}}+\frac{1}{z} .
$$

Inserting this into (3.17), we find that for $f \notin \mathrm{E}_{m}^{1}$,

$$
\begin{aligned}
\log L\left(1, \operatorname{sym}^{m} f\right)= & \sum_{p \leq z} \sum_{0 \leq j \leq m} \log \left(1-\frac{\alpha_{f}(p)^{m-2 j}}{p}\right)^{-1} \\
& +O\left(\frac{1}{(\log k)^{\min \left\{\delta_{0}, \delta_{2}-\delta_{0}\right\}}}+\frac{1}{z}\right)
\end{aligned}
$$

which will give the required result.

$3^{\circ}$ The case $y_{2} \leq z<y_{1}$. We truncate the tail as in (3.17), and use the estimate of the second case. Thus it remains to evaluate

$$
R_{2}\left(\operatorname{sym}^{m} f\right):=-\sum_{z<p \leq y_{1}} \sum_{0 \leq j \leq m} \log \left(1-\frac{\alpha_{f}(p)^{m-2 j}}{p}\right)
$$


Let us take

$P_{i}:=2^{i-1} z, \quad Q_{i}:=\min \left\{2^{i} z, y_{1}\right\}, \quad \mathrm{E}_{m}^{2}:=\mathrm{H}_{k, \mathrm{sym}^{m}}^{-}(1 ; \eta) \cup \bigcup_{i \ll \log _{2} k} \mathrm{E}_{m}^{2}\left(P_{i}, Q_{i}\right)$.

By Lemma 3.3, we have

$$
\left|\mathrm{E}_{m}^{2}\right| \ll k^{31 \eta}+k e^{-(\theta(m)+2) z^{\delta_{3}}} \log _{2} k \ll k e^{-\theta(m) z^{\delta_{3}}}
$$

and

$$
\begin{aligned}
R_{2}\left(\operatorname{sym}^{m} f\right) & \ll \sum_{i \ll \log _{2} k}\left|T_{\text {sym }^{m} f}\left(P_{i}, Q_{i}\right)\right|+\frac{1}{z} \\
& \ll \sum_{i \ll \log _{2} k} \frac{1}{\left(2^{i-1} z\right)^{\delta_{4}}}+\frac{1}{z} \ll \frac{1}{z^{\delta_{4}}}
\end{aligned}
$$

for all $f \notin \mathrm{E}_{m}^{2}$.

Finally, define $\mathrm{E}_{k}^{*}:=\mathrm{E}_{m}^{1} \cup \mathrm{E}_{m}^{2}$. Then we have

$$
\left|\mathrm{E}_{k}^{*}\right| \ll k e^{-\theta(m) z^{\delta_{3}}} .
$$

In view of (3.19) and (3.18), we derive that

$$
\begin{aligned}
\log L\left(1, \operatorname{sym}^{m} f\right)= & \sum_{p \leq z} \sum_{0 \leq j \leq m} \log \left(1-\frac{\alpha_{f}(p)^{m-2 j}}{p}\right)^{-1} \\
& +O\left(\frac{1}{(\log k)^{\delta_{5}}}+\frac{1}{z^{\delta_{4}}}\right)
\end{aligned}
$$

for $f \in \mathrm{H}_{k}^{*}(1) \backslash \mathrm{E}_{k}^{*}$, where $\delta_{5}:=\min \left\{\delta_{0}, \delta_{1}, \delta_{2}-\delta_{0}\right\}$. Obviously this is equivalent to our required result. The proof of Theorem 1.1 is thus complete.

4. Proof of Corollary 1.2. By Theorem 1.1 with the choice of

$$
z=\log k, \quad \theta_{1}=1 / 2-\varepsilon, \quad \theta_{2}=\theta_{3}=\varepsilon,
$$

there is a subset $\mathrm{E}_{k}^{*}$ of $\mathrm{H}_{k}^{*}(1)$ such that $\left|\mathrm{E}_{k}^{*}\right| \ll k e^{-(\log k)^{1 / 2-\varepsilon}}$ and

$$
L\left(1, \operatorname{sym}^{m} f\right)=\left\{1+O\left(\frac{1}{(\log k)^{\varepsilon}}\right)\right\} \prod_{p \leq z} \prod_{0 \leq j \leq m}\left(1-\frac{\alpha_{f}(p)^{m-2 j}}{p}\right)^{-1}
$$

for each $f \in \mathrm{H}_{k}^{*}(1) \backslash \mathrm{E}_{k}^{*}$. In view of (1.4) and the prime number theorem, it follows that

$$
\begin{aligned}
L\left(1, \operatorname{sym}^{m} f\right) & \leq\left\{1+O\left(\frac{1}{(\log k)^{\varepsilon}}\right)\right\} \prod_{p \leq z}\left(1-\frac{1}{p}\right)^{-(m+1)} \\
& =\left\{1+O\left(\frac{1}{(\log k)^{\varepsilon}}\right)\right\}\left(e^{\gamma} \log _{2} k\right)^{m+1}
\end{aligned}
$$


for all $f \in \mathrm{H}_{k}^{*}(1) \backslash \mathrm{E}_{k}^{*}$. This proves the upper bound result in Corollary 1.2 and one can treat the lower bound in the same way.

\section{References}

[1] S. Chowla, Improvement of a theorem of Linnik and Walfisz, Proc. London Math. Soc. 50 (1949), 423-429.

[2] S. Chowla and P. Erdoos, A theorem on the distribution of the values of L-functions, J. Indian Math. Soc. 15 (1951), 11-18.

[3] J. Cogdell and P. Michel, On the complex moments of symmetric power L-functions at $s=1$, Int. Math. Res. Not. 2004, no. 31, 1561-1618.

[4] P. Deligne, La conjecture de Weil, I, II, Inst. Hautes Études Sci. Publ. Math. 48 (1974), 273-308, 52 (1981), 313-428.

[5] W. Duke and E. Kowalski, A problem of Linnik for elliptic curves and mean-value estimates for automorphic representations, with an appendix by Dinakar Ramakrishnan, Invent. Math. 139 (2000), 1-39.

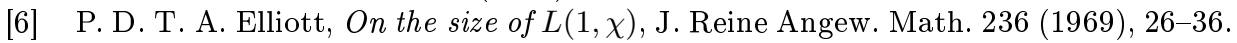

[7] - The distribution of the quadratic class number, Litovsk. Mat. Sb. 10 (1970), 189-197.

[8] - On the mean value of $f(p)$, Proc. London Math. Soc. (3) 21 (1970), 28-96.

[9] A. Granville and K. Soundararajan, The distribution of values of $L\left(1, \chi_{d}\right)$, Geom. Funct. Anal. 13 (2003), 992-1028.

[10] L. Habsieger and E. Royer, L-functions of automorphic forms and combinatorics: Dyck paths, Ann. Inst. Fourier (Grenoble) 54 (2004), 2105-2141.

[11] J. Hoffstein and P. Lockhart, Coefficients of Maass forms and the Siegel zero, Ann. of Math. 140 (1994), 161-181.

[12] H. Iwaniec, W. Luo and P. Sarnak, Low lying zeros of families of L-functions, Inst. Hautes Études Sci. Publ. Math. 91 (2000), 55-131.

[13] E. Kowalski and P. Michel, The analytic rank of $J_{0}(q)$ and zeros of automorphic L-functions, Duke Math. J. 100 (1999), 503-542.

[14] Y.-K. Lau and J. Wu, A density theorem on automorphic L-functions and some applications, Trans. Amer. Math. Soc. 358 (2006), 441-472.

[15] J. E. Littlewood, On the class number of the corpus $P(\sqrt{-k})$, Proc. London Math. Soc. 27 (1928), 358-372.

[16] W. Luo, Values of symmetric square L-functions at 1, J. Reine Angew. Math. 506 (1999), 215-235.

[17] H. L. Montgomery and R. C. Vaughan, Extreme values of Dirichlet L-functions at 1 , in: Number Theory in Progress, Vol. 2 (Zakopane-Kościelisko, 1997), K. Györy et al. (eds.), de Gruyter, Berlin, 1999, 1039-1052.

[18] E. Royer, Statistique de la variable aléatoire $L\left(1, \mathrm{sym}^{2} f\right)$, Math. Ann. 321 (2001), 667-687.

[19] - Interprétation combinatoire des moments négatifs des valeurs de fonctions $L$ au bord de la bande critique, Ann. Sci. École Norm. Sup. (4) 36 (2003), 601-620.

[20] E. Royer et J. Wu, Taille des valeurs de fonctions $L$ de carrés symétriques au bord de la bande critique, Rev. Mat. Iberoamericana 21 (2005), 263-312.

[21] - - - Central values, values at the edge of the critical strip of symmetric power $L$-functions and Hecke eigenvalues, preprint.

[22] G. Tenenbaum, Introduction to Analytic and Probabilistic Number Theory, Cambridge Stud. Adv. Math. 46, Cambridge Univ. Press, Cambridge, 1995. 
[23] R. C. Vaughan, Small values of Dirichlet L-functions at 1, in: Analytic Number Theory, Vol. 2 (Allerton Park, IL, 1995), Progr. Math. 139, Birkhäuser Boston, $1996,755-766$.

Department of Mathematics

Institut Élie Cartan

The University of Hong Kong

UMR 7502 UHP CNRS INRIA

Pokfulam Road, Hong Kong

Université Henri Poincaré (Nancy 1)

E-mail: yklau@maths.hku.hk

54506 Vandœuvre-lès-Nancy, France

E-mail: wujie@iecn.u-nancy.fr

Received on 18.7.2005

and in revised form on 7.11.2006 